\title{
The Levels of Inhibitory Cytokines in the Serum of Patients with Hepatitis B and C
}

\author{
Ainor Rohmah ${ }^{1}$, Sigit Setyawan ${ }^{2}$, Tri Nugraha Susilawati ${ }^{3}$ \\ ${ }^{1}$ Undergraduate Program in Medicine, Faculty of Medicine, Sebelas Maret University, Surakarta, Indonesia \\ ${ }^{2}$ Department of Parasitology, Faculty of Medicine, Sebelas Maret University, Surakarta, Indonesia \\ ${ }^{3}$ Department of Microbiology, Faculty of Medicine, Sebelas Maret University, Surakarta, Indonesia. E-mail: tri.susilawati@staff.uns.ac.id
}

\begin{abstract}
Hepatitis B and C Viruses (HBV dan HCV) can cause acute or chronic hepatitis that may develop into fibrosis, cirrhosis, and hepatocarcinoma. Previous studies have reported that hepatocyte damage is mainly due to overactive immune responses rather than viral infection. Cytokines are essential mediators in the immune response. This study aimed to determine the correlation between the levels of serum inhibitory cytokines, i.e., IL-4, IL-10, and TGF- $\beta$, and the development of liver disease in patients with hepatitis B and C. The levels of serum IL-4, IL-10, and TGF- $\beta$ from 58 patients with hepatitis B or hepatitis $C$ were determined by ELISA. The progression of liver disease is inferred from the levels of serum transaminases and the degree of liver fibrosis. Data were analyzed using the Spearman correlation test with a $p$-value of $<0.05$ is considered statistically significant. This study showed no correlation between the levels of serum IL-4, IL-10, and TGF- $\beta$ and the development of liver disease in patients with hepatitis B and C $(p>0.05)$. Therefore, cytokine testing using ELISA was unable to replace liver biopsy to assess liver disease progression in patients with hepatitis $B$ and $C$.
\end{abstract}

Keywords: IL-4, IL-10, TGF- $\beta$, disease progression, hepatitis B, hepatitis C

\section{INTRODUCTION}

Hepatitis $B$ and hepatitis $C$ remain public health problems and are possible to be fatal. ${ }^{1}$ Data from the World Health Organization (WHO) in 2018 showed that 257 and 170 million people had been infected with HBV and HCV infection, respectively. ${ }^{2,3}$ These infections cause more than one million deaths per year, and most of them are caused by cirrhosis and hepatocarcinoma. ${ }^{2,3} \mathrm{~A}$ total of 19 million and 2.5 million people in Indonesia suffer from hepatitis B and $C .5$ The preventive measures and therapeutic attempts for hepatitis $B$ and $C$ remain challenging. Vaccines are only available for hepatitis $B$, and the currently available treatments can only slow down the progression of chronic liver diseases. The only treatment for the final stage of liver failure is liver transplantation; however, its implementation is relatively difficult even in developed countries. ${ }^{2,3}$

Hepatitis B viruses and HCV infection can cause acute or chronic liver diseases that may progress to fibrosis, cirrhosis, and liver cancer. Previous studies have reported that the viruses themselves do not cause the damage of hepatocytes due to their non-cytopathic (inability to damage cells) properties. ${ }^{6.7}$ The possible cause of hepatocytes damage in HBV and HCV infection is the body's immune responses toward infected hepatocytes to minimize the numbers of intracellular viruses. ${ }^{6.8}$ the damage of This immune-associated cell is similar to that in autoimmune hepatitis of which is diagnosed with a combination of biochemical (such as LKM-1 autoantibodies, anti-smooth muscle antibodies, and ANA), immunological (e.g., elevated serum immunoglobulin), and histological testing with the exclusion of other causes of liver disease (i.e., viral hepatitis). ${ }^{9,10}$

Cytokines are proteins released by immune cells that serve as essential mediators in the immune response. Some cytokines such as interleukin-4 (IL-4), IL-10, and Transforming Growth Factor-Beta (TGF- $\beta$ ) can inhibit or regulate the immune response itself. ${ }^{8}$ It has been reported that IL-10 is closely related to disease progression and can be used as an early predictive marker for the development of acute exacerbation of chronic hepatitis B. ${ }^{11}$ Similarly, IL-4 is an important cytokine in predicting advanced fibrosis in patients with hepatitis C. In chronic hepatitis $B$, the progression from liver fibrosis into liver cirrhosis can be modified by changing the levels of serum cytokines such as TGF- $\beta$, IL- 6 , and IL- $17 .{ }^{12}$ This study aimed to determine the correlation 
between serum levels of cytokines IL-4, IL-10, and TGF- $\beta$ with the disease progression in patients with hepatitis $B$ and hepatitis $C$.

\section{METHODS}

This study was analytical observational research with a cross-sectional design. The study involved a total of 58 patients with hepatitis $B$ or hepatitis $C$ who were admitted to Dr. Moewardi Hospital, Surakarta, Indonesia, from October to December 2017. The collected serum samples were stored at $-800 \mathrm{C}$ until further use. IL-4, IL-10, and TGF- $\beta 1$ in patients' serum were measured using the Enzyme-Linked Immunosorbent Assay (ELISA). The progression of hepatitis $B$ and $C$ disease is characterized by developing the inflammatory phases (acute and chronic infection), fibrosis, cirrhosis, and hepatocarcinoma. ${ }^{13}$ The disease progression was evaluated based on the levels of liver transaminases and the results of fibroscan. ${ }^{14}$ The data on the patient's disease progression were collected from medical records. Data analysis was performed using the Spearman correlation test, and a $p$-value of $<0.05$ was considered statistically significant. The study protocol had been approved by the Health Research Ethics Committee at Dr. Moewardi Hospital (548/VIII/HREC/2017).

\section{RESULTS AND DISCUSSIONS}

Table 1 shows that most participants (36\%) were early elderly (46-55 years old). Most patients had chronic hepatitis $B(n=38 ; 66 \%)$ and chronic hepatitis C $(n=9 ; 15 \%)$. A small proportion of subjects had advanced hepatitis characterized by hepatic cirrhosis $(n=7 ; 12 \%)$, and a few of the patients developed hepatocarcinoma $(n=3 ; 5 \%)$. Most of the study participants had normal levels of liver transaminases. The level of fibrosis could not be determined in most subjects since liver biopsy is an invasive procedure. In this study, only 14 out of 58 (24\%) subjects had fibroscan data, and from these available data, while most patients had severe fibrosis with cirrhosis (F4).

The progression of hepatitis can be assessed through the patient's clinical condition, laboratory tests of liver function, radiology (ultrasound or fibroscan), and pathological anatomy. Liver biopsy is the gold standard to determine the severity of the liver disease. However, this method is invasive and relatively expensive. Therefore, the levels of serum cytokines were tested using ELISA because it was expected that the cytokines could be used instead of liver biopsy as the markers to determine liver disease progression.
Table 1. The characteristics of subjects

\begin{tabular}{lc}
\hline Description & $\mathbf{n = 5 8}$ \\
\hline Age (in years) & \\
Early adults (26-35) & $6(10 \%)$ \\
Late adult (36-45) & $10(17 \%)$ \\
Early elderly (46-55) & $21(36 \%)$ \\
Late elderly (56-65) & $13(23 \%)$ \\
$\quad$ Seniors (>65) & $8(14 \%)$ \\
Diagnosis & \\
Acute hepatitis B & $1(2 \%)$ \\
Chronic hepatitis B & $38(66 \%)$ \\
Chronic hepatitis C & $9(15 \%)$ \\
Cirrhosis hepatic & $7(12 \%)$ \\
Hepatocarcinoma & $3(5 \%)$ \\
ALT $^{15}$ & \\
Normal (5-35 U/L) & $37(64 \%)$ \\
Mild increase (1-3x normal) & $10(17 \%)$ \\
Moderate increase (3-20x normal) & $11(19 \%)$ \\
Severe increase (> 20x normal) & $0(0 \%)$ \\
AST $^{15}$ & \\
Normal (5-35 U/L) & $27(47 \%)$ \\
Mild increase (1-3x normal) & $24(41 \%)$ \\
Moderate increase (3-20x normal) & $7(12 \%)$ \\
Severe increase (>20x normal) & $0(0 \%)$ \\
Fibrosis $^{14,16}$ & \\
< F2 & $2(14 \%)$ \\
F2-F3 & $5(36 \%)$ \\
F4 & $7(50 \%)$ \\
\hline
\end{tabular}

In this study, the results of ELISA were divided into three groups; the lower quartile, the middle quartile, and the upper quartile (Table 2). The predominant levels of serum IL-4 were in the lower quartile group $(n=23 ; 40 \%), I L-10$ in the middle quartile group $(n=27 ; 48 \%)$, and TGF- $\beta$ in the middle quartile group $(n=28 ; 48 \%)$.

Table 2. The levels of IL-4, IL-10, and TGF- $\beta$ measured by ELISA

\begin{tabular}{lc}
\hline \multicolumn{1}{c}{ Description } & n (\%) \\
\hline IL-4 & $23(40 \%)$ \\
Lower quartile $(\leq 0.21 \mathrm{pg} / \mathrm{mL})$ & $14(24 \%)$ \\
$\quad$ Middle quartile $(0.22-0.23 \mathrm{pg} / \mathrm{mL})$ & $21(36 \%)$ \\
$\quad$ Upper quartile $(\geq 0.24 \mathrm{pg} / \mathrm{mL})$ & \\
IL-10 & $16(27 \%)$ \\
$\quad$ Lower quartile $(\leq 0.305 \mathrm{pg} / \mathrm{mL})$ & $27(48 \%)$ \\
$\quad$ Middle quartile $(0.306-0.52 \mathrm{pg} / \mathrm{mL})$ & $15(25 \%)$ \\
$\quad$ Upper quartile $(\geq 0.53 \mathrm{pg} / \mathrm{mL})$ & \\
TGF- $\boldsymbol{\beta}$ & $15(26 \%)$ \\
$\quad$ Lower quartile $(\leq 2.88 \mathrm{pg} / \mathrm{mL})$ & $28(48 \%)$ \\
$\quad$ Middle quartile $(2.89-4.15 \mathrm{pg} / \mathrm{mL})$ & $15(26 \%)$ \\
$\quad$ Upper quartile $(\geq 4.16 \mathrm{pg} / \mathrm{mL})$ & $\mathbf{5 8}$ \\
Total &
\end{tabular}


The liver disease progression in this study was determined by evaluating the levels of ALT, AST, and METAVIR scores ( $F 0=$ no fibrosis; $F 1=$ portal fibrosis without septa; F2=portal fibrosis with septa; F3=advanced fibrosis without cirrhosis; F4=cirrhosis). ${ }^{16}$ The results from the Spearman correlation test showed a weak correlation between the patient's age and the cytokines and between the disease progression and the cytokines $(r<0.1)$. Table 3 shows the direction of the relationship and the significance levels among variables tested. The correlation between patient's age and the stories of cytokines tested was not statistically significant $(p>0.05)$. Similarly, the correlation between disease progression and the levels of cytokines tested was not statistically significant ( $p>0.05$ ). The direction of the relationship varied among variables tested, and this was not further discussed since the correlation was weak and not statistically significant.

Table 3. The significance levels ( $p$-values) and the direction of the relationship among variables obtained from the Spearman correlation test

\begin{tabular}{lccc}
\hline & IL-4 & IL-10 & TGF- $\boldsymbol{\beta}$ \\
\hline Age & $0.83(+)$ & $0.46(+)$ & $0.50(-)$ \\
Disease progression & $0.37(+)$ & $0.52(-)$ & $0.93(-)$ \\
\hline
\end{tabular}

$(+)$ : positive correlation; (-): negative correlation

The majority of participants in this present study were 46-55 years old or early elderly, similar to the previous report. ${ }^{17}$ The most probable reason for this finding was that the subjects were asymptomatic at the early onset of infection, thus delaying the diagnosis. Previous studies reported that the productive age group (15-64 years old) had more risk factors for $\mathrm{HBV}$ and $\mathrm{HCV}^{5,18}$ The risk factors for $\mathrm{HBV}$ infection include having sex with HBV infected person, using interchangeable equipment (such as syringes, toothbrushes, razors, or medical devices), babies born to infected mothers, and medical workers who are exposed to the blood of HBVinfected persons. ${ }^{19}$ The risk factors for HCV infection include use of the intravenous drug, alternate use of unsterile needles, tattoos and piercings equipment, having multiple sex partners, and HIV infection. ${ }^{20}$

Most of the participants in this study had chronic hepatitis B. A previous study reported that the prevalence of hepatitis $B$ is very high in the Southeast Asia region, and there are nearly $25 \%$ cases of chronic liver diseases such as fibrosis, cirrhosis, and hepatocarcinoma. ${ }^{21}$ The prevalence of hepatitis B in Indonesia generally reaches $7.1 \%$ of the population of Indonesia, indicating that Indonesia is categorized as a moderate hepatitis B endemic area. However, several provinces in Indonesia have variations in the prevalence rate of hepatitis $B$, showing two to three times higher rates. ${ }^{4,5}$

Most of our study participants had normal ALT and AST levels. Normal ALT and AST levels can be found naturally in chronic hepatitis; for example, in patients in the immunotolerance phase where the immune system is tolerant to viruses in the body, and there is no inflammation in the liver, indicating relatively normal ALT and AST levels. ${ }^{6,22}$ Normal ALT and AST levels can also be due to regular treatment in hepatitis B patients. ${ }^{23}$ The correlation between certain cytokine levels and the progression of liver disease regardless of the patient's medication history was the only thing discussed in this study, indicating a limitation of this study.

Fibroscan results from our data showed that most participants in this study had severe fibrosis with cirrhosis. Globally, nearly $30 \%$ of adults with chronic $\mathrm{HBV}$ infection have cirrhosis, and the risk is higher in older age. ${ }^{24}$ Elevated HBV DNA levels in chronic hepatitis also increase the risk of developing cirrhosis. ${ }^{25}$ A previous study suggested that HBV DNA levels of greater than 2,000 IU/mL were strongly correlated with the development of cirrhosis. ${ }^{26}$

This study showed that the levels of serum IL-4, IL-10, and TGF- $\beta$ were mostly in the lower or middle quartile category, which was similar to those in healthy people. Generally, cytokine levels in healthy individuals are undetectable or detectable in small or low amounts. ${ }^{27}$ The similarity of cytokine levels between patients and healthy people can be caused by various factors, such as internal factors (i.e., the phase of the patient's disease and the immune status of the subjects) or external factors (i.e., sample storage condition and test method).

Samples in this study had been frozen and thawed repeatedly, which might affect the results of ELISA. A previous study reported that repeated clotting and thawing of serum samples could change the levels of serum IL-4, IL-6, IL-8, and IL-9 measured by using ELISA. ${ }^{28}$ Another study reported differences in the levels of Vascular Endothelial Growth Factor (VEFG) as measured from specimens by repeated freeze-thawing. Circulating VEFG levels were found to be elevated in the freeze-thaw cycle samples. ${ }^{29}$

The length of sample storage may also affect cytokine levels. The study samples were collected in late 2017, and ELISA tests were carried out in early 2020. IL-1 $\alpha$, IL-1 $\beta$, IL-5, IL-6, and IL-10 were reported to degrade up to $50 \%$ in the storage of frozen serum samples at $-80^{\circ} \mathrm{C}$ for two to three years, and repeated 
freezing and thawing of the samples increased the levels of cytokines IL-4 and TNF- $\alpha$ compared to baseline values. ${ }^{30}$ Repeated clotting and thawing should be avoided in serum testing. If the direct assay of samples is complex, the serum should not be left at room temperature or refrigerated but frozen and stored at $-80^{\circ} \mathrm{C}$ instead, as soon as samples are collected from subjects. ${ }^{31}$

The progression of liver disease in this study was evaluated based on the levels of liver transaminases and the degree of fibrosis. It has been reported that ALT level is an essential indicator of hepatocellular damage. ${ }^{32}$ Previous studies reported mixed results regarding the correlation between the levels of cytokines and liver transaminases. A previous study reported a significant correlation between IL-10 and ALT in chronic hepatitis $B .{ }^{33}$ Other studies have shown a significant correlation between the levels of IL-4 and IL-10 with the levels of ALT and AST in hepatitis. ${ }^{29,34}$ In addition, a positive correlation between TGF- $\beta$ levels with ALT and AST had been reported in patients with liver cirrhosis. ${ }^{35}$ Another study in chronic hepatitis B said that there was no significant correlation between IL-10 level and AST levels. ${ }^{36}$ Another study reported no significant correlation between the levels of TGF- $\beta$ and ALT in chronic hepatitis C. Drug therapy might have an effect. ${ }^{32}$

In this present study, the degree of liver fibrosis was categorized into three groups based on the METAVIR scores, i.e., less than F2, F2 to F3, and F4. Previous studies reported that elevated TGF- $\beta$ significantly increased the risk of developing liver cirrhosis in chronic hepatitis. The mechanism of TGF- $\beta$-induced fibrogenesis is estimated by activating Human Stellate Cells (HSC) in the liver, which then produces extracellular matrix proteins involved in the development of fibrosis. ${ }^{12} \mathrm{HCV}$ can also interact with stellate cells that release TGF- $\beta$ and induce fibrogenesis in these cells, increasing the production of pro-collagen $\alpha 1$ (I) and pro-collagen $\alpha 1$ (III) and decreasing the expression of fibrinolytic Matrix Metalloproteinases (MMP). ${ }^{37}$ In addition, IL-4 activates stellate cells (HSC) and enhances collagen production in HSC by signal transduction and activation of the 6-related pathway of transcription. IL-4 also activates collagen synthesis by non-parenchymal hepatic cells. ${ }^{37}$ Another study reported a significant increase of IL-4 levels in hepatitis C patients with severe fibrosis. ${ }^{37}$ In chronic hepatitis infection, a high serum IL-10 can lead to an immunosuppressive environment produced by several cells such as dendritic cells in the liver. This immunosuppressive environment plays a protective role in hepatitis infection associated with the development of liver fibrosis. ${ }^{38}$ Other studies showed a significant correlation between IL-10 and fibrosis scores in chronic hepatitis $\mathrm{B}^{12}$ This study showed that there was no significant correlation between the levels of serum IL- 4 , IL-10, and TGF- $\beta$ with disease progression in hepatitis $B$ and hepatitis $C$ patients. The number of samples in our study group might influence the results of this study. It was suggested that further research should have a minimum of 30 samples in each group. ${ }^{39}$

\section{CONCLUSIONS AND SUGGESTIONS}

There was no significant correlation between the levels of serum IL-4, IL-10, and TGF- $\beta$ and hepatitis progression. Therefore, cytokine testing using ELISA cannot replace liver biopsy for monitoring liver disease progression in hepatitis $B$ and $C$. It was recommended to perform cytokine testing using freshly collected serum samples. If fresh samples are not possible, it is necessary to consider factors that may affect the results, such as the length of the sample storage and repeated thawing and freezing. Further research with more subjects can better describe the correlation between serum cytokines and disease progression in hepatitis B and C.

\section{ACKNOWLEDGMENT}

The authors thank Dr. Triyanta Yuli Pramana for his help in collecting samples and Alfin Titian Permata for her help during laboratory work. Sebelas Maret University provided funding support for this research (No: 516/UN27.21/PP/2019).

\section{REFERENCES}

1. https://www.who.int/health-topics/hepatitis \#tab=tab_1 (accessed 6 Jan, 2021)

2. https://www.who.int/news-room/fact-sheets/ detail/hepatitis-b (accessed 11 Des, 2020)

3. https://www.who.int/news-room/fact-sheets/detail/ hepatitis-c (accessed 11 Des, 2020)

4. http://cevhap.org/index.php/resources/cevhap -publications/item/29-situation-analysis-of-viral -hepatitis-in-indonesia-a-po (accessed 5 Jan, 2021)

5. Muldoon D. Epidemiology of hepatitis B and C in Republic of Indonesia. Euroasian Journal of Hepato-Gastroenterology, 2017; 7: 55-59.

6. Mauss B, Rockstroh S, Wedemeyer. Hepatology a clinical textbook. 10 ${ }^{\text {th }}$ Ed., Germany, GILEAD, 2020; 44-47.

7. Khan S, Bhargava A, Pathak N, Maudar KK, et al. 
Circulating biomarkers and their possible role in pathogenesis of chronic hepatitis $B$ and $C$ viral infections. Indian Journal of Clinical Biochemistry, 2011; 26(2): 161-168.

8. Zdravkovic N, Rosic M, Lutovac M, Zdravkovic V. Physiology and Pathology of Cytokine: Commercial Production and Medical Use. Intech. 2018; 10.5772/intechopen.72200.

9. Tiniakos DG, Brain JG, Bury YA. Role of histopathology in autoimmune hepatitis. Dig Dis, 2015; 33(2): 53-64.

10. Czaja AJ. Diagnosis and Management of Autoimmune Hepatitis: Current Status and Future Directions. Gut Liver, 2016; 10(2): 177-203.

11. Magda R, Anna W, Alicja S, Tomasz R, Piotr S, et al. Genetic variation in IL-10 influences the progression of hepatitis $B$ infection. International Journal of Infectious Diseases, 2020; 96: 260-265.

12. Cai GF, Wang ZT, Yao J, Liu K, Wang Z, et al. The correlation between serum cytokine levels and liver cirrhosis in chronic hepatitis B patients: A meta-analysis. Archives of Hepatitis Research, 2018; 4(1): 6-12.

13. https://liverfoundation.org/for-patients/about-the -liver/the-progression-of-liver-disease/(accessed 9 Nov, 2020)

14. https://apps.who.int/iris/bitstream/10665/ 111747/1/9789241548755_eng.pdf. (accessed 6 Aug, 2020)

15. Stein SM. BOH'S pharmacy practice manual: A guide to the clinical experience. $3^{\text {rd }}$ Ed., Philadelphia, Lippincott Williams \& Wilkins, 2010; 117-196.

16. Liao R, Fu YP, Wang T, Deng ZG, Li DW, et al. Metavir and FIB-4 scores are associated with patient prognosis after curative hepatectomy in hepatitis $B$ virus-related hepatocellular carcinoma: $A$ retrospective cohort study at two centers in China. Oncotarget, 2017; 8(1): 1774-1787.

17. Pokharel N, Bhandari D, Jha B. Pattern of hepatitis B and $C$ infections among patients attending a tertiary care hospital in Kathmandu, Nepal. Journal of Institute of Medicine, 2017; 40.

18. Neoh H, Saimin S, Jamal R. Seroprevalence of hepatitis $B$ and $C$ in healthy Malaysian adults: A preliminary report. Journal of Biomedicine and Translational Research, 2019; 5: 23.

19. https://www.cdc.gov/hepatitis/hbv/hbvfaq.htm. (accessed 18 June, 2020).

20. https://www.cdc.gov/hepatitis/hcv/hcvfaq.htm. (accessed 18 June, 2020)

21. Soe KP, Pan-ngum W, Nontprasert A, Kittitrakul C, Oam N, et al. Awareness, knowledge, and practice for hepatitis B infection in Southeast Asia: a cross-sectional study. J Infect Dev Ctries, 2019; 13: 656-664.

22. Viky NRM, Puspa W, Bagus S. AST, ALT and albumin level in chronic hepatitis B patients with and without complications of cirrhosis and hepatocellular carcinoma. Indonesian Journal of Clinical Pathology and Medical Laboratory, 2020; 26(3): 344-349.
23. https://www.cancertherapyadvisor.com/home /decision-support-in-medicine/hospital-medicine/ elevated-liver-enzymes-hepatitis/. (accessed 30 May, 2021).

24. https://www.infectiousdiseaseadvisor. com/home/advisor-channels/hepatitis-advisor/high - prevalence-of-cirrhosis-in-adults-with-chronic-hbv -at-a-safety-net -hospital/ (accessed 13 Feb, 2021).

25. Liu C, Wang L, Xie H, Zhang L, Wang B, et al. The relationship between serum hepatitis $B$ virus DNA level and liver histology in patients with chronic HBV infection. PLoS One, 2018; 13(11): e0206060.

26. European Association for the Study of the Liver. Clinical practice guidelines on the management of hepatitis B virus infection. Journal of Hepatology, 2017; 67(2): 370-398.

27. Danlu W, Trinh LD, Bruce PB, David RW. Long-term measurements of human inflammatory cytokines reveal complex baseline variations between individuals. The American Journal of Pathology, 2017; 187(12): 2620-26.

28. Lugos MD, Polit UY, Damen JG. A study on the impact of repeated freeze-thaw cycles on the serum cytokine Enzyme-Linked Immunosorbent Assay (ELISA). Int. J. Med. Lab. Res, 2018; 3(2): 29-36.

29. Agrawal L, Engel KB, Greytak SR, Moore HM. Understanding pre-analytical variables and their effects on clinical biomarkers of oncology and immunotherapy. Semin Cancer Biol. 2018; 52(Pt 2): 26-38.

30. Samantha S, Janina K, Seth G, Lubna P. Thermal stability of cytokines: A review. Cytokine. 2020; 125: 154829.

31. González DR, González DÁ, Sayago A, Fernández RÁ. Recommendations and best practices for standardizing the pre-analytical processing of blood and urine samples in metabolomics. Metabolites, 2020; 10(6): 229.

32. Albanian WK, Fathy SA, Ibrahim RA, Hegazy MGA. Assessment of interleukin 17 and transforming growth factor-beta 1 in hepatitis $C$ patients with disease progression. Trop Biomed, 2020; 37(4): 1093-1104.

33. Hosseini KSH, Nejatollahi F, Davarpanah MA. Serum levels of interleukin-4, interleukin-10 and interferon-y in patients with chronic hepatitis B infection. Hepat Mon, 2018; 18(4): e60377.

34. Gu Y, Lian Y, Gu L, Chen L, Li X, et al. Correlations between cytokines produced by $\mathrm{T}$-cells and clinical-virological characteristics in untreated chronic hepatitis B patients. BMC Infect Dis, 2019; 19(1): 216.

35. Tian F, Liu Y, Gao J, Yang N, Shang X, et al. Study on the association between TGF- $\beta 1$ and liver fibrosis in patients with hepatic cystic echinococcosis. Experimental and Therapeutic Medicine. 2020; 19: 1275-80.

36. Ming DS, Lin ZZ, Qiu XD. Analysis of IL-6, IL-10, IL-17 levels in the blood of patients with chronic hepatitis $B$. Int J Clin Exp Med, 2016; 9(6): 9437-9442.

37. Batsaikhan B, Lu MY, Yeh ML, Huang CI, Huang CF, et al. Elevated interleukin-4 levels predicted advanced 
fibrosis in chronic hepatitis C. JCMA, 2019; 277-281.

38. Li H, Huang MH, Jiang JD, Peng ZG. Hepatitis C: From inflammatory pathogenesis to anti-inflammatory/ hepatoprotective therapy. World Journal of
Gastroenterology, 2018; 24(47): 5297-5311

39. Strunk KK, Mwavita M. Design and analysis in educational research: ANOVA designs in SPSS ${ }^{\circ}, 1^{\text {st }}$ Ed., London, Routledge, 2020; 24-26. 